\title{
Positive solutions for a semipositone problem involving nonlocal operator
}

\author{
G.A. AfrouzI(*) - N.T. ChUnG(**) - S. SHAKERI(***)
}

ABSTRACT - In this article, we are interested in the existence of positive solutions for the following Kirchhoff type problems

$$
\left\{\begin{array}{l}
-M\left(\int_{\Omega}|\nabla u|^{p} d x\right) \operatorname{div}\left(|\nabla u|^{p-2} \nabla u\right)=\lambda a(x) f(u)-\mu \text { in } \Omega, \\
u=0 \text { on } x \in \partial \Omega
\end{array}\right.
$$

where $\Omega$ is a bounded smooth domain of $R^{N}, 1<p<N, M: R_{0}^{+} \rightarrow R^{+}$is a continuous and increasing function, $\lambda, \mu$ are two positive parameters, $a \in C(\bar{\Omega})$, $a(x) \geq a_{0}>0$, and $f$ is a $C^{1}([0, \infty))$ function such that $f(0)=0, f(t)>0$ for all $0<t<t_{0}$ and $f(t) \leq 0$ for all $t \geq t_{0}$, where $t_{0}>0$.

Mathematics Subject Classification (2010). 35D05, 35J60.

KEYWORDS. Kirchhoff type problems; semipositone; positive solution; sub and supersolutions.

\section{Introduction}

In this paper, we are interested in the existence of positive solutions for a class of Kirchhoff type problems of the form

(*) Indirizzo dell'A.: Department of Mathematics, Faculty of Mathematical Sciences, University of Mazandaran, Babolsar, Iran.

E-mail: afrouzi@umz.ac.ir

(**) Indirizzo dell'A.: Dept. Science Management \& International Cooperation, Quang Binh University, 312 Ly Thuong Kiet, Dong Hoi, Quang Binh, Vietnam.

E-mail: ntchung82@yahoo.com

(***) Indirizzo dell'A.: Department of Mathematics, Faculty of Mathematical Sciences, University of Mazandaran, Babolsar, Iran.

E-mail: s.shakeri@umz.ac.ir 
(1)

$$
\left\{\begin{array}{l}
-M\left(\int_{\Omega}|\nabla u|^{p} d x\right) \operatorname{div}\left(|\nabla u|^{p-2} \nabla u\right)=\lambda a(x) f(u)-\mu \text { in } \Omega, \\
u=0 \text { on } x \in \partial \Omega
\end{array}\right.
$$

where $\Omega$ is a bounded smooth domain of $R^{N}, 1<p<N, \lambda, \mu$ are two positive parameters, the functions $M, a, f$ satisfy the following conditions:

$\left(H_{1}\right) M: R_{0}^{+} \rightarrow R^{+}$is a continuous and increasing function and $M(t) \geq m_{0}>0$ for all $t \in R_{0}^{+}$, where $R_{0}^{+}:=[0,+\infty) ;$

$\left(H_{2}\right) \quad a \in C(\bar{\Omega})$ and $a(x) \geq a_{0}>0$;

$\left(H_{3}\right) f \in C^{1}([0, \infty))$ and $f(0)=0$,

$\left(H_{4}\right)$ There exists $t_{0}>0$ such that $f(t)>0$ for all $0<t<t_{0}$ and $f(t) \leq 0$ for all $t \geq t_{0}$.

Since the first equation in (1) contains an integral over $\Omega$, it is no longer a pointwise identity; therefore it is often called nonlocal problem. This problem models several physical and biological systems, where $u$ describes a process which depends on the average of itself, such as the population density, see [4]. Moreover, problem (1) is related to the stationary version of the Kirchhoff equation

$$
\rho \frac{\partial^{2} u}{\partial t^{2}}-\left(\frac{P_{0}}{h}+\frac{E}{2 L} \int_{0}^{L}\left|\frac{\partial u}{\partial x}\right|^{2} d x\right) \frac{\partial^{2} u}{\partial x^{2}}=0
$$

presented by Kirchhoff in 1883, see [9]. This equation is an extension of the classical d'Alembert's wave equation by considering the effects of the changes in the length of the string during the vibrations. The parameters in (2) have the following meanings: $L$ is the length of the string, $h$ is the area of the cross-section, $E$ is the Young modulus of the material, $\rho$ is the mass density, and $P_{0}$ is the initial tension.

In recent years, problems involving Kirchhoff type operators have been studied in many papers, we refer to $[1,2,3,5,10,13,14,15]$ in which the authors have used variational method and topological method to get the existence of solutions for (1). In this paper, motivated by the ideas introduced in [11] and the properties of Kirchhoff type operators in $[6,7,8]$, we study problem (1) in the semipositone case, see the condition $\left(H_{3}\right)$. Using the sub- and supersolutions techniques, we establish positive constants $\mu^{*}=\mu^{*}\left(\Omega, t_{0}\right)$ and $\lambda_{*}=$ $\lambda_{*}\left(\Omega, t_{0}, \mu\right)$ such that problem (1) has a positive solution when $\mu \leq \mu^{*}$ 
and $\lambda \geq \lambda_{*}$. Our result in this note improves the previous one [11] in which $M(t) \equiv 1, a(x) \equiv 1$. It is clear that our situation in this present paper is different from [1] in which we studied the problem with one parameter. To our best knowledge, this is a new research topic for nonlocal problems, see $[1,8]$.

Our main result is given by the following theorem.

THEOREM 1.1. Under the conditions $\left(H_{1}\right)-\left(H_{4}\right)$, there exist positive constants $\mu^{*}=\mu^{*}\left(\Omega, t_{0}\right)$ and $\lambda_{*}=\lambda_{*}\left(\Omega, t_{0}, \mu\right)$ such that problem (1) has a positive solution when $\mu \leq \mu^{*}$ and $\lambda \geq \lambda_{*}$.

\section{Preliminaries}

In this paper, we denote $W_{0}^{1, p}(\Omega)$, the completion of $C_{0}^{\infty}(\Omega)$, with respect to the norm

$$
\|u\|=\left(\int_{\Omega}|\nabla u|^{p} d x\right)^{\frac{1}{p}} .
$$

In order to precisely state our main result we first consider the following eigenvalue problem for the $p$-Laplace operator $-\Delta_{p} u$, see [11]:

$$
\left\{\begin{array}{l}
-\Delta_{p} u=\lambda|u|^{p-2} u \quad \text { in } \Omega, \\
u=0 \quad \text { on } x \in \partial \Omega .
\end{array}\right.
$$

Let $\phi_{1} \in C^{1}(\bar{\Omega})$ be the eigenfunction corresponding to the first eigenvalue $\lambda_{1}$ of (3) such that $\phi_{1}>0$ in $\Omega$ and $\left\|\phi_{1}\right\|_{\infty}=1$. It can be shown that $\frac{\partial \phi_{1}}{\partial \eta}<0$ on $\partial \Omega$ and hence, depending on $\Omega$, there exist positive constants $m, \delta, \sigma$ such that

$$
\left\{\begin{array}{l}
\left|\nabla \phi_{1}\right|^{p}-\lambda_{1} \phi_{1}^{p} \geq m \quad \text { on } \bar{\Omega}_{\delta}, \\
\phi_{1} \geq \sigma \quad \text { on } x \in \Omega \backslash \bar{\Omega}_{\delta},
\end{array}\right.
$$

where $\bar{\Omega}_{\delta}:=\{x \in \Omega: d(x, \partial \Omega) \leq \delta\}$.

We will also consider the unique solution $e \in W_{0}^{1, p}(\Omega)$ of the boundary value problem

$$
\left\{\begin{array}{l}
-\Delta_{p} e=1 \quad \text { in } \Omega, \\
e=0 \quad \text { on } x \in \partial \Omega
\end{array}\right.
$$



to discuss our result. It is known that $e>0$ in $\Omega$ and $\frac{\partial e}{\partial \eta}<0$
on $\partial \Omega$.

We will prove our result by using the method of sub- and supersolutions, we refer the readers to a recent paper [8] on the topic. A function $\psi$ is said to be a subsolution of problem (1) if it is in $W^{1, p}(\Omega) \cap C^{0}(\bar{\Omega})$ such that $\psi=0$ on $\partial \Omega$ and satisfies

$$
\begin{aligned}
& M\left(\int_{\Omega}|\nabla \psi|^{p} d x\right) \int_{\Omega}|\nabla \psi|^{p-2} \nabla \psi \cdot \nabla w d x \leq \\
& \qquad \int_{\Omega}(\lambda a(x) f(\psi)-\mu) w d x, \quad \forall w \in W,
\end{aligned}
$$

where $W:=\left\{w \in C_{0}^{\infty}(\Omega): w \geq 0\right.$ in $\left.\Omega\right\}$. A function $\phi \in W^{1, p}(\Omega) \cap C^{0}(\bar{\Omega})$ is said to be a supersolution if $\phi=0$ on $\partial \Omega$ and satisfies

$$
\begin{aligned}
& M\left(\int_{\Omega}|\nabla \psi|^{p} d x\right) \int_{\Omega}|\nabla \psi|^{p-2} \nabla \psi \cdot \nabla w d x \geq \\
& \qquad \int_{\Omega}(\lambda a(x) f(\psi)-\mu) w d x, \quad \forall w \in W .
\end{aligned}
$$

The following result plays an important role in our arguments. The readers may consult the papers $[1,6,7]$ for details.

Lemma 2.1. Assume that $M: R_{0}^{+} \rightarrow R^{+}$satisfies the condition $\left(H_{1}\right)$. If the functions $u, v \in W_{0}^{1, p}(\Omega)$ satisfies

$$
\begin{gathered}
M\left(\int_{\Omega}|\nabla u|^{p} d x\right) \int_{\Omega}|\nabla u|^{p-2} \nabla u \cdot \nabla \varphi d x \leq \\
M\left(\int_{\Omega}|\nabla v|^{p} d x\right) \int_{\Omega}|\nabla v|^{p-2} \nabla v \cdot \nabla \varphi d x
\end{gathered}
$$

for all $\varphi \in W_{0}^{1, p}(\Omega), \varphi \geq 0$, then $u \leq v$ in $\Omega$.

From Lemma 2.1 we obtain the following basic principle of the sub- and supersolutions method. 
Positive solutions for a semipositone problem involving nonlocal operator 29

THEOREM 2.2 (See $[1,8]$ ). Let $M: R_{0}^{+} \rightarrow R^{+}$be a function satisfying the condition $\left(H_{1}\right)$. Assume that $f$ satisfies the subcritical growth condition

$$
|f(x, t)| \leq C\left(1+|t|^{q-1}\right), \quad \forall x \in \Omega, \quad \forall t \in R,
$$

where $1<q<p^{*}$, and the function $f(x, t)$ is nondecreasing in $t \in R$. If there exist a subsolution $\underline{u} \in W^{1, p}(\Omega)$ and a supersolution $\bar{u} \in$ $W^{1, p}(\Omega)$ of problem (1), then (1) has a minimal solution $u_{*}$ and $a$ maximal solution $u^{*}$ in the order interval $\left[u_{*}, u^{*}\right]$, i.e., $\underline{u} \leq u_{*} \leq$ $u^{*} \leq \bar{u}$ and if $u$ is any solution of (1) such that $\underline{u} \leq u \leq \bar{u}$, then $u_{*} \leq u \leq u^{*}$.

In the practice problems, it is often known that the subsolution $\underline{u}$ and the supersolution $\bar{u}$ are of $L^{\infty}(\Omega)$, so the restriction on the growth condition of $f$ is needless. Hence, the following theorem is more suitable for our framework.

THEOREM 2.3 (See $[1,8]$ ). Let $M: R_{0}^{+} \rightarrow R^{+}$be a function satisfying the condition $\left(H_{1}\right)$. Assume that $\underline{u}, \bar{u} \in W^{1, p}(\Omega) \cap L^{\infty}(\Omega)$ are a subsolution and a supersolution of problem (1) such that $\underline{u} \leq \bar{u}$ in $\Omega$. If $f \in C(\bar{\Omega} \times R, R)$ is nondecreasing in $t \in\left[\inf _{\Omega} \underline{u}, \sup _{\Omega} \bar{u}\right]$ then the conclusion of Theorem 2.2 is valid.

\section{Proof of the main result}

Let $\lambda_{1}, \phi_{1}, \delta, m, \sigma$ and $\Omega_{\delta}$ be as described in Section 2 . We now construct our positive subsolution. Let

$$
\psi:=\frac{p-1}{p} t_{0} \phi_{1}^{\frac{p}{p-1}}
$$

It should be noticed that $\|\psi\|_{\infty}<t_{0}$. Then $\nabla \psi=t_{0} \phi_{1}^{\frac{1}{p-1}} \nabla \phi_{1}$ and $\psi$ will be a subsolution if

(9) $M\left(\int_{\Omega}|\nabla \psi|^{p} d x\right) \int_{\Omega}|\nabla \psi|^{p-2} \nabla \psi \cdot \nabla w d x \leq$

$$
\int_{\Omega}[\lambda a(x) f(\psi)-\mu] w d x, \quad \forall w \in W
$$


But

$$
\begin{aligned}
& M\left(\int_{\Omega}|\nabla \psi|^{p} d x\right) \int_{\Omega}|\nabla \psi|^{p-2} \nabla \psi \cdot \nabla w d x \\
& =t_{0}^{p-1} M\left(\int_{\Omega}|\nabla \psi|^{p} d x\right) \int_{\Omega}\left|\nabla \phi_{1}\right|^{p-2} \phi_{1} \nabla \phi_{1} \cdot \nabla w d x \\
(10) & =t_{0}^{p-1} M\left(\int_{\Omega}|\nabla \psi|^{p} d x\right)\left[\int_{\Omega}\left|\nabla \phi_{1}\right|^{p-2} \nabla \phi_{1} \cdot \nabla\left(\phi_{1} w\right) d x-\int\left|\nabla \phi_{1}\right|^{p} w d x\right] \\
& =r t_{0}^{p-1} M\left(\int_{\Omega}|\nabla \psi|^{p} d x\right) \int_{\Omega}\left[\lambda_{1} \phi_{1}^{p}-\left|\nabla \phi_{1}\right|^{p}\right] w d x \\
& \leq m_{0} t_{0}^{p-1} \int_{\Omega}\left[\lambda_{1} \phi_{1}^{p}-\left|\nabla \phi_{1}\right|^{p}\right] w d x .
\end{aligned}
$$

Now, by (4), we have in $\bar{\Omega}_{\delta}$,

$$
m_{0} t_{0}^{p-1}\left[\lambda_{1} \phi_{1}^{p}-\left|\nabla \phi_{1}\right|^{p}\right] \leq-m_{0} m t_{0}^{p-1}
$$

Hence, if $\mu \leq \mu^{*}:=m_{0} m t_{0}^{p-1}$ then it implies by $\left(H_{2}\right)$ and $f(\psi) \geq 0$ that

$$
m_{0} t_{0}^{p-1}\left[\lambda_{1} \phi_{1}^{p}-\left|\nabla \phi_{1}\right|^{p}\right] \leq \lambda a(x) f(\psi)-\mu \text { in } \bar{\Omega}_{\delta} .
$$

Next in $\Omega \backslash \bar{\Omega}_{\delta}$, we have

$$
m_{0} t_{0}^{p-1}\left[\lambda_{1} \phi_{1}^{p}-\left|\nabla \phi_{1}\right|^{p}\right] \leq \lambda_{1} m_{0} t_{0}^{p-1}
$$

while

$$
\lambda a(x) f(\psi)-\mu \geq \lambda a_{0} \alpha-\mu,
$$

where

$$
\alpha=\inf \left\{f(s): \frac{p-1}{p} t_{0} \sigma^{\frac{p}{p-1}} \leq s \leq \frac{p-1}{p} t_{0}\right\} .
$$

Hence, if $\lambda \geq \lambda_{*}=\frac{\lambda_{1} m_{0} t_{0}^{p-1}+\mu}{a_{0} \alpha}$ then in $\Omega \backslash \bar{\Omega}_{\delta}$,

$$
m_{0} t_{0}^{p-1}\left[\lambda_{1} \phi_{1}^{p}-\left|\nabla \phi_{1}\right|^{p}\right] \leq \lambda a(x) f(\psi)-\mu .
$$

Hence, if $\mu \leq \mu^{*}$ and $\lambda \geq \lambda_{*}$ then (9) is satisfied and $\psi$ is a subsolution. 
Positive solutions for a semipositone problem involving nonlocal operator 31

Next, we construct a supersolution $\phi$ such that $\phi \geq \psi$. Let $\phi:=L e$, where $e$ is the solution of problem (5). Then, using the condition $\left(H_{1}\right), \phi$ will be a supersolution of problem (1) if

$$
\begin{aligned}
& M\left(\int_{\Omega}|\nabla \phi|^{p} d x\right) \int_{\Omega}|\nabla \phi|^{p-2} \nabla \phi \cdot \nabla w d x \\
& =L^{p-1} M\left(\int_{\Omega}|\nabla \phi|^{p} d x\right) \int_{\Omega}|\nabla e|^{p-2} \nabla e \cdot \nabla w d x \\
& =L^{p-1} M\left(\int_{\Omega}|\nabla \phi|^{p} d x\right) \int_{\Omega} w d x \\
& \geq m_{0} L^{p-1} \int_{\Omega} w d x \\
& \geq \int_{\Omega}[\lambda a(x) f(\psi)-\mu] w d x, \quad \forall w \in W .
\end{aligned}
$$

But

$$
m_{0} L^{p-1} \int_{\Omega} w d x \geq \int_{\Omega}[\lambda a(x) f(\psi)-\mu] w d x \quad \forall w \in W
$$

provided that

$$
m_{0} L^{p-1} \geq \lambda \cdot\|a\|_{\infty} \sup _{t \in\left[0, t_{0}\right]} f(t) .
$$

That is, if $L \geq\left(\frac{\lambda \cdot\|a\|_{\infty} \sup _{t \in\left[0, t_{0}\right]} f(t)}{m_{0}}\right)^{\frac{1}{p-1}}$ then (12) is satisfied and $\phi$ is a supersolution of problem (1). Since $e>0$ in $\Omega$ and $\frac{\partial e}{\partial n}<0$ on $\partial \Omega$, we can choose $L$ large enough so that $\phi \geq \psi$ is also satisfied. Therefore, Theorem 1.1 is proved.

\section{REFERENCES}

[1] G.A. Afrouzi - N.T. Chung - S. Shakeri, Existence of positive solutions for Kirchhoff type equations, Electron. J. Differential Equations 2013, no. 180,8 pp. 
[2] C.O. Alves - F.J.S.A. CorRÊA - T.M. MA, Positive solutions for a quasilinear elliptic equation of Kirchhoff type, Comput. Math. Appl. 49 (2005), pp. 85-93.

[3] A. BensediK - M. BoucheKIF, On an elliptic equation of Kirchhoff-type with a potential asymptotically linear at infinity, Math. Comput. Modelling $\mathbf{4 9}$ (2009), pp. 1089-1096.

[4] M. CHIPOT - B. Lovat, Some remarks on nonlocal elliptic and parabolic problems, Nonlinear Anal. 30 (1997), no. 7, pp. 4619-4627.

[5] F.J.S.A. CorrêA - G.M. Figueiredo, On an elliptic equation of p-Kirchhoff type via variational methods, Bull. Austral. Math. Soc. 74 (2006), pp. 263-277.

[6] G. DAI, Three solutions for a nonlocal Dirichlet boundary value problem involving the $p(x)$-Laplacian, Appl. Anal. 92 (2013), no. 1, pp. 191-210.

[7] G. DAI - R. MA, Solutions for a p(x)-Kirchhoff type equation with Neumann boundary data, Nonlinear Anal. Real World Appl. 12 (2011), pp. 2666-2680.

[8] X. HAN - G. DAI, On the sub-supersolution method for $p(x)$-Kirchhoff type equations, J. Inequal. Appl. (2012), 11 pp.

[9] G. KirchHoff, Mechanik, Teubner, Leipzig, Germany, 1883.

[10] T.F. MA, Remarks on an elliptic equation of Kirchhoff type, Nonlinear Anal. 63 (2005), pp. 1967-1977.

[11] S. Oruganti - R. ShivaJI, Existence results for classes of p-Laplacian semipositone equations, Bound. Value Probl. (2006), Art. ID 87483, 7 pp.

[12] K. Perera - Z. Zhang, Nontrivial solutions of Kirchhoff-type problems via the Yang index, J. Differential Equations 221 (2006), no. 1, pp. 246-255.

[13] B. RICCERI, On an elliptic Kirchhoff-type problem depending on two parameters, J. Global Optim. 46 (2010), no. 4, pp. 543-549.

[14] J.J. SUn - C.L. TANG, Existence and multiplicity of solutions for Kirchhoff type equations, Nonlinear Anal. 74 (2011), pp. 1212-1222.

[15] M.H. YANG - Z.Q. HAN, Existence and multiplicity results for Kirchhoff type problems with four-superlinear potentials, Appl. Anal. 91 (2012), no. 11, pp. 2045-2055.

[16] Z. ZhAng - K. Perera, Sign changing solutions of Kirchhoff type problems via invariant sets of descent flow, J. Math. Anal. Appl. 317 (2006), no. 2, pp. $456-463$.

Manoscritto pervenuto in redazione il 21 Agosto 2013. 